\title{
Estudo das características epidemiológicas e clínicas de pacientes portadores do vírus da imunodeficiência humana em Pronto Atendimento do Hospital das Clínicas da Universidade Federal de Minas Gerais
}

\author{
Study on the clinical and epidemiological characteristics of patients with \\ human immunodeficiency virus at the walk-in service of the teaching \\ Hospital of the Federal University of Minas Gerais
}

Cecilia Gómez Ravetti ${ }^{1,2}$ e Ênio Roberto Pietra Pedroso ${ }^{1,2}$

\begin{abstract}
RESUMO
Este trabalho objetiva a análise prospectiva das características clínicas e epidemiológicas que levam os pacientes adultos com HIV/AIDS a procurarem atendimento clínico de urgência em Pronto Atendimento do Hospital das Clínicas da Universidade Federal de Minas Gerais. Noventa e nove pacientes perfizeram 118 internações. A idade foi em média 39,4 anos. A relação homem e mulher foi de 1,35:1. 0 tempo desde o diagnóstico até a admissão situou-se de forma mais freqüente entre $0-5$ anos em 40,4\% dos casos. A terapêtica anti-retroviral era usada regularmente em $56,8 \%$ das admissões. A contagem de linfócitos $\mathrm{T}$ CD $4^{+}$foi inferior a 200 células $/ \mathrm{mm}^{3} \mathrm{em} 45,7 \%$ dos pacientes. As queixas mais frequientes foram aumento da temperatura corpórea, diarréia, tosse e dispnéia. 0 aparelho respiratório foi o mais acometido. As doenças oportunistas mais freqüentes foram pneumocistose, pneumonia comunitária, síndrome diarréica, e candidiase oral. A demanda de internações de pacientes com HIV representou 2,8\% das admissões, com tempo médio de permanência hospitalar de 4,6 dias. Os pacientes possuíam, em sua maioria, contagem de linfócitos TCD4+ baixa, quase metade não usava a terapia anti-retroviral altamente eficaz. Houve tendência à feminização. As doenças relacionadas à AIDS continuam sendo as mais freqüentes no nosso meio.
\end{abstract}

Palavras-chaves: Vírus da imunodeficiência humana. Síndrome da imunodeficiência humana. Urgências. Emergências. Doenças oportunistas.

\section{ABSTRACT}

This study had the aim of prospectively analyzing the clinical and epidemiological characteristics that lead adult patients with HIV/AIDS to seek urgent medical care in the walk-in service of the teaching hospital of the Federal University of Minas Gerais. Over a one-year period, all admissions of adult patients with HIV were evaluated. There were 118 admissions involving 99 patients. Their mean age was 39.4 years. The male-to-female ratio was 1.35:1. The length of time from diagnosis to admission was most frequently $0-5$ years ( $40.4 \%$ of the cases). Antiretroviral therapy was being regularly used among $56.8 \%$ of the admissions. The T CD $4^{+}$lymphocyte count was less than 200 cells $/ \mathrm{mm}^{3}$ in $45.7 \%$ of the patients. The most frequent complaints were increased body temperature, diarrhea, coughing and dyspnea. The respiratory tract was the region most affected. The most common opportunistic diseases were pneumocystosis, community-acquired pneumonia, diarrheal syndrome and oral candidiasis. The demand for admissions among HIV patients represented $2.8 \%$ of all admissions, with an average length of stay of 4.6 days. Most of the patients had low T CD $4+$ lymphocyte counts, and almost half of them were not taking highly active antiretroviral therapy. There was a tendency towards feminization. AIDS-related diseases continue to be the most frequent occurrences within our setting.

Key-words: Human immunodeficiency virus. Acquired immunodeficiency syndrome. Urgency. Emergency. Opportunistic diseases.

A pandemia da índrome da imunodeficiência humana (AIDS) tornou-se, nos últimos quinze anos, uma entidade nosológica de convivência, crônica, desde que abordada de forma adequada ${ }^{1416}$. A incidência das infecções oportunistas diminuiu desde a introdução da terapia anti-retroviral (TARV) altamente eficaz, levando a uma

1. Pronto Atendimento, Hospital das Clínicas, Universidade Federal de Minas Gerais, Belo Horizonte, MG. 2. Curso de Pós Graduação em Ciências da Saúde, Infectologia e Medicina Tropical, Universidade Federal de Minas Gerais, Belo Horizonte, MG. Endereço para correspondência: Dra Cecilia Gómez Ravetti. Rua Sagitário 320/01, Santa Lúcia, 30360-230 Belo Horizonte, MG.

Tel: 5531 3024-9413; 5531 9959-6555

e-mail: ceciliag.ravetti@gmail.com

Recebido para publicação em 15/05/2008

Aceito em 05/03/2009 redução nas taxas de morbidade e mortalidade associadas à infecção pelo vírus da imunodeficiência humana (HIV) ${ }^{81011}$.

Assim, na era pós-TARV, as internações hospitalares decorrem, principalmente, devido às doenças relacionadas com infecções oportunistas, complicações relacionadas à medicação, e às doenças não relacionadas ao HIV ${ }^{257}$. Houve redução no número de internações hospitalares, especialmente daquelas devido a infecções oportunistas ${ }^{5}$, e aumento significativo na contagem dos linfócitos TCD4 à admissão hospitalar ${ }^{15}$. Alguns trabalhos evidenciaram, entretanto, maior prevalência de internações por estas infecções ${ }^{1}$.

No Brasil, observou-se redução no percentual de internações de pacientes com HIV no Sistema Único de Saúde, porém com 
menor número entre os pacientes em uso de TARV ${ }^{4}$, com grande variação nas diferentes regiões ${ }^{313}$.

Este trabalho objetiva a análise prospectiva das características clínicas e epidemiológicas que levam os pacientes com HIV a procurarem Pronto Atendimento Clínico de Referência na região metropolitana de Belo Horizonte, que tem cerca de cinco milhões de pessoas.

\section{MATERIAL E MÉTODOS}

Foram estudados, entre junho de 2006 e maio de 2007, todos os pacientes adultos infectados pelo HIV, admitidos consecutivamente no Pronto Atendimento do Hospital das Clínicas (PA-HC) da Universidade Federal de Minas Gerais (UFMG). Os dados foram obtidos por intermédio da análise dos prontuários no Pronto Atendimento, e no Serviço de Arquivo Médico (SAME), retrospectivamente, quando houve necessidade de complementar informação. Foram excluídos os pacientes menores de 18 anos e com permanência por menos de 24 horas.

Este estudo foi aprovado pela Comissão de Ética em Pesquisa (COEP) da UFMG, mediante o Parecer número 292/06.

Estatística. Baseou-se na análise descritiva univariada das características epidemiológicas e clínicas dos pacientes à admissão. 0 pacote estatístico utilizado nas análises foi o SPSS 12.0 para Windows. Foi utilizado o teste de Shapiro-Wilk na verificação da normalidade das variáveis contínuas. 0 Teste t de Student foi utilizado para amostras independentes. Foi utilizado o teste não paramétrico de Mann-Whitney para comparação de medianas. 0 teste Exato de Fisher foi utilizado para comparação de proporções. 0 nível de significância considerado foi igual 0,05.

0 registro de internações no Pronto Atendimento foi realizado por intermédio de informações obtidas da ficha específica gerada no sistema do próprio Pronto Atendimento.

\section{RESULTADOS}

Foram analisados 99 pacientes, que perfizeram 118 internações. Os pacientes tinham em média 39,4 anos ( $\mathrm{DP}=9,1)$. A maioria dos pacientes era do sexo masculino $(57,6 \%)$, com relação homem: mulher 1,35/1. Os pacientes eram procedentes, em sua maioria, de Belo Horizonte. Em relação à etnia, 27,3\% eram negros, 23,2\% brancos e $17,2 \%$ pardos. De acordo com o estado civil, 50,5\% eram solteiros, 22,2\% casados e 5,1\% viúvos (Tabela 1).

A forma de transmissão não foi informada na maioria dos pacientes, e, entre os que a informou, a heterossexual foi a predominante com 18,2\% (Tabela 1). 0 tempo de diagnóstico de infecção pelo HIV e a internação foi entre 0 a 5 anos em $40,4 \%$, de 5 a 10 anos em 22,2\% e mais de 10 anos em 19,2\% (Tabela 1).

Em relação ao acompanhamento hospitalar prévio à internação, 55,1\% dos casos eram acompanhados em algum Centro de Referência, ou possuíam, pelo menos, dois atendimentos clínicos anuais de rotina, sendo, então, considerado adequado. A terapia anti-retroviral era usada regularmente em 56,8\% dos pacientes admitidos, segundo relato dos mesmos, e não usada por $34,7 \%$. A quimioprofilaxia para doenças oportunistas era usada por $29,7 \%$ dos pacientes admitidos e não usada por $48,3 \%$ dos casos (Tabela 2).

No que diz respeito à contagem dos linfócitos T CD4+ no momento ou na época mais próxima da sua admissão, observou-se que 45,7\% possuíam menos de 200 células $/ \mathrm{mm}^{3}$ (Tabela 2).

Não foi detectada associação significativa do valor da contagem dos linfócitos T CD4+ em função do desfecho óbito $(\mathrm{p}=0,294)$, e o uso de profilaxia para infecções oportunistas em função do mesmo desfecho $(\mathrm{p}=1,0)$.

As principais queixas à admissão hospitalar e os principais sistemas acometidos encontram-se na Tabela 3.

TABELA 1

Variáveis sócio-demográficas dos pacientes portadores de HIV/AIDS internados em Pronto Atendimento.

\begin{tabular}{|c|c|c|c|c|c|}
\hline Variável & Número & Percentagem & Variável & Número & Percentagem \\
\hline \multicolumn{3}{|l|}{ Sexo } & \multicolumn{3}{|l|}{ Cidade de origem } \\
\hline feminino & 42 & 42,4 & Belo Horizonte & 63 & 63,6 \\
\hline \multirow[t]{3}{*}{ masculino } & \multirow[t]{3}{*}{57} & \multirow[t]{3}{*}{57,6} & interior Minas Gerais & 29 & 29,3 \\
\hline & & & outro Estado & 3 & 3,0 \\
\hline & & & não informado & 4 & 4,0 \\
\hline Total & 99 & 100,0 & Total & 99 & 100,0 \\
\hline \multicolumn{3}{|l|}{$\overline{\text { Cor }}$} & \multicolumn{3}{|l|}{ Modo mais provável de transmissão } \\
\hline branca & 23 & 23,2 & heterossexual & 18 & 18,2 \\
\hline negra & 27 & 27,3 & homosexual & 2 & 2,0 \\
\hline parda & 17 & 17,2 & sangüínea & 4 & 4,0 \\
\hline \multirow[t]{2}{*}{ não informado } & \multirow[t]{2}{*}{32} & \multirow[t]{2}{*}{32,3} & vertical & 1 & 1,0 \\
\hline & & & não informado & 74 & 74,7 \\
\hline Total & 99 & 100,0 & Total & 99 & 100,0 \\
\hline \multicolumn{3}{|l|}{ Estado civil } & \multicolumn{3}{|l|}{ Tempo de diagnóstico (anos) } \\
\hline solteiro & 50 & 50,5 & $0-5$ & 40 & 40,4 \\
\hline casado & 22 & 22,2 & $5-10$ & 22 & 22,2 \\
\hline viúvo & 5 & 5,1 & $>10$ & 19 & 19,2 \\
\hline não informado & 22 & 22,2 & não informado & 18 & 18,2 \\
\hline Total & 99 & 100,0 & Total & 99 & 100,0 \\
\hline
\end{tabular}


TABELA 2

Variáveis relacionadas com o tratamento dos pacientes portadores do HIV/AIDS.

\begin{tabular}{lcr}
\hline Variável & Número & Percentagem \\
\hline Controle ambulatorial & & \\
adequado & 65 & 55,1 \\
não adequado & 35 & 29,7 \\
não informado & 18 & 15,3 \\
\hline Total & 118 & 100,0 \\
\hline
\end{tabular}

Terapia anti-retroviral (3 drogas)

\begin{tabular}{lrr} 
não & 41 & 34,7 \\
sim & 67 & 56,8 \\
não informado & 10 & 8,5 \\
\hline Total & 118 & 100,0
\end{tabular}

Profilaxia das infecções oportunistas

\begin{tabular}{lrr} 
não & 57 & 48,3 \\
sim & 35 & 29,7 \\
não informado & 26 & 22,0 \\
\hline Total & 118 & 100,0 \\
\hline Contagem dos linfócitos TCD4 & & \\
$<100$ & 34 & 28,8 \\
$101-200$ & 20 & 16,9 \\
$201-350$ & 10 & 8,5 \\
$351-500$ & 15 & 12,7 \\
$\geq 500$ & 1 & 0,8 \\
não informado & 31 & 26,3 \\
\hline Total & 118 & 100,0 \\
\hline
\end{tabular}

TABELA 3

Sintomas encontrados e sistemas mais freqüentemente acometidos no momento da admissão.

\begin{tabular}{lccccc}
\hline & \multicolumn{2}{c}{ Sim } & & \multicolumn{2}{c}{ Total } \\
\cline { 2 - 3 } \cline { 5 - 6 } Variável & número & percentagem & & número & percentagem \\
\hline Sintomatologia principal & & & & & \\
febre & 34 & 28,8 & & 118 & 100,0 \\
tosse & 25 & 21,2 & & 118 & 100,0 \\
dispnéia & 16 & 13,6 & & 118 & 100,0 \\
cefaléia & 36 & 30,5 & & 118 & 100,0 \\
diarréia & 16 & 13,6 & & 118 & 100,0 \\
$\quad$ náuseas/vômitos & & & & \\
Sistemas acometidos & 43 & 36,4 & & 118 & 100,0 \\
$\quad$ respiratório & 42 & 35,6 & & 118 & 100,0 \\
gastrintestinal & 25 & 21,2 & & 118 & 100,0 \\
neurológico & 12 & 10,2 & & 118 & 100,0 \\
geniturinário & 12 & 10,2 & & 118 & 100,0 \\
hematológico & 5 & 4,2 & 118 & 100,0 \\
cardiovascular & 11 & 9,1 & 118 & 100,0 \\
outros & & & &
\end{tabular}

Não foi detectada associação significativa entre os sistemas acometidos em função do desfecho óbito.

Os diagnósticos nosológicos mais freqüentes foram pneumocistose $(17,8 \%)$, pneumonia comunitária (16,9\%), diarréia $(16,1 \%)$, candidiase oral $(10,2 \%)$, neurotoxoplasmose $(9,3 \%)$, e neutropenia febril $(8,4 \%)$.
TABELA 4

Variáveis relacionadas com a internação hospitalar.

\begin{tabular}{|c|c|c|}
\hline Variável & Número & Percentagem \\
\hline \multicolumn{3}{|l|}{ Admissão hospitalar } \\
\hline sala observação & 102 & 86,4 \\
\hline sala de emergência & 13 & 11,0 \\
\hline não informado & 3 & 2,5 \\
\hline Total & 118 & 100,0 \\
\hline \multicolumn{3}{|l|}{ Uso de ventilação mecânica } \\
\hline não & 112 & 94,9 \\
\hline $\operatorname{sim}$ & 3 & 2,5 \\
\hline não informado & 3 & 2,5 \\
\hline Total & 118 & 100,0 \\
\hline \multicolumn{3}{|l|}{ Desfecho inicial } \\
\hline alta hospitalar & 59 & 50,0 \\
\hline internação enfermaria & 39 & 33,1 \\
\hline óbito & 4 & 3,4 \\
\hline transferência outro hospital & 3 & 2,5 \\
\hline não informado & 13 & 11,0 \\
\hline Total & 118 & 100,0 \\
\hline \multicolumn{3}{|c|}{ Desfecho após internação em enfermaria } \\
\hline alta hospitalar & 26 & 66,7 \\
\hline óbito & 5 & 12,8 \\
\hline transferência outro hospital & 1 & 2,6 \\
\hline não informado & 7 & 17,9 \\
\hline Total & 39 & 100,0 \\
\hline
\end{tabular}

Dos pacientes hospitalizados, $102(86,4 \%)$ foram admitidos na Sala de Observação, e 13 (11\%) na Sala de Emergência, sendo que 2,5\% precisaram ventilação mecânica (Tabela 4). 0 desfecho após internação no PA-HC/UFMG encontra-se na Tabela 4. 0 óbito foi constatado durante a observação no Pronto Atendimento em quatro pacientes e durante internação na enfermaria em cinco, representando uma mortalidade de 7,6\%.

As 118 admissões de pacientes com HIV representaram 2,83\% do total de internações realizadas no Pronto Atendimento do Hospital das Clínicas da UFMG no período estudado.

0 tempo de permanência nesta sala foi, em média, de 4,6 dias.

Não foi encontrada diferença estatisticamente significativa entre idade em anos e tempo de permanência em dias, em função do desfecho óbito, nem associação significativa do desfecho óbito em função do gênero, etnia, modo de transmissão, controle ambulatorial, terapia anti-retroviral e tempo de diagnóstico.

\section{DISCUSSÃO}

0 motivo que leva o paciente portador do HIV à internação hospitalar tem mudado desde a introdução da HAART, de forma dinâmica ${ }^{1012} 1315$.

Este trabalho evidenciou que no Pronto Atendimento do Hospital das Clínicas da UFMG, Centro de Referência para emergências clínicas da região metropolitana de Belo Horizonte, 
houve tendência de equivalência entre os dois gêneros (relação homem/mulher 1,35/1), desde a prevalência inicial expressiva do sexo masculino, revelando o seu caráter de feminizaçã $0^{213}$.

A forma de transmissão do HIV não foi determinada na maioria dos pacientes $(74,7 \%)$, entretanto, entre os que relataram como se infectaram, prevaleceu a transmissão sexual, especialmente a heterossexual. Esses dados revelam tendência de redução da participação na epidemia pelo HIV no Brasil da transmissão pelas vias homossexual e vertical desde a introdução de controles estritos de doadores, de medidas de profilaxia em pessoas de risco, e de seguimento rigoroso em grávidas com a administração, quando necessária, de profilaxia anti-retroviral ${ }^{9}$.

Observou-se que um número elevado de pacientes, $42 \%$, tinha o diagnóstico de infecção pelo HIV realizado há mais de 5 anos, caracterizando a cronicidade da evolução da doença, que parece relacionado, especialmente, ao papel da terapia anti-retroviral, da profilaxia de infecções oportunistas, à redução da mortalidade e ao aumento da sobrevida ${ }^{128}$. Porém, não foi constatada diferença estatisticamente significativa entre o tempo de diagnóstico da doença e a evolução ao óbito.

0 acompanhamento destes pacientes em centros de referência ou pelo menos dois atendimentos anuais foi observado em somente $55,1 \%$, revelando, ainda um cuidado precário.

A contagem de linfócitos TCD 4+, nas vezes em que foi identificada, revelou que em $45,7 \%$ dos casos era inferior a 200 células $/ \mathrm{mm}^{3}$ e inferior a 350 células em $54,2 \%$ dos pacientes, demonstrando, então, a grande procura por atendimento nos pacientes com estádio mais avançado da infecção pelo HIV. A quimioprofilaxia regular foi anotada em $43 \%$ dos pacientes com linfócitos TCD4+ inferiores a 200, e que perfaziam 45\% dentre todos os pacientes. Estes dados revelam que, apesar do Brasil possuir um sistema altamente qualificado em todos os níveis da atenção à saúde para o paciente com AIDS, os pacientes possuem baixa aderência terapêutica, o que coloca em risco toda a estratégia de controle da disseminação do HIV. Estes dados indicam, ainda, a necessidade de conscientização sobre a relação entre saúde-doença, reforça a importância e 0 valor da educação para a saúde. Em estudo recentemente realizado, observou-se aumento na taxa de interrupção da terapia anti-retroviral quando os pacientes eram submetidos exclusivamente a acompanhamento ambulatorial, em comparação ao acompanhamento domiciliar, porém sem diferença estatisticamente significativa ${ }^{17}$.

O tempo médio de permanência no Pronto Atendimento foi de 4,6 dias, valor que ultrapassa o tempo usualmente esperado para a observação em Sala de Emergência, que é de, no máximo, de 24 a 48 horas. 0 objetivo do Pronto Atendimento é a recepção do paciente com necessidade urgente de identificação de alguma alteração fisiopatológica súbita, grave, com risco de provocar limitação funcional principal ou morte. Esta média evidencia a situação falimentar do sistema de urgência do SUS que não consegue resolver os vários níveis da atenção médica, transferindo para a urgência a demanda por atenção à saúde como um todo e não consegue providenciar, em tempo adequado, o atendimento correto e necessário. Em 42\% dos casos aqui estudados a observação inicial no Pronto Atendimento foi seguida da necessidade de outro nível de atenção médica, representado por enfermaria de nível secundário ou terciário (Sala de emergência), ou para outra instituição de internação hospitalar em decorrência de ausência de disponibilidade de leitos hospitalares no Hospital das Clínicas. Estes dados apontam para a necessidade de redimensionamento da atenção hospitalar para os pacientes com AIDS, com expansão de leitos e adequação de condições de trabalho adequadas.

0 óbito foi constatado em nove pacientes durante a internação, quatro no Pronto Atendimento e cinco após a internação hospitalar, o que representa mortalidade de 7,6\%. A contagem de linfócitos era inferior a 100 em cinco pacientes, e entre 350 e 500 células/ $/ \mathrm{mm}^{3} \mathrm{em}$ um perfazendo assim a relação de 5:1, entretanto, sem diferença estatisticamente significativa $(\mathrm{p}=0,294)$.

A demanda de internação no Pronto Atendimento do Hospital das Clínicas da UFMG para os portadores do HIV situou-se em $2,8 \%$ de todas as admissões. A queixa principal que levou os pacientes a procurarem atendimento foi 0 aumento da temperatura corpórea, seguida pela diarréia, tosse e dispnéia, cefaléia, náuseas e vômitos. 0 aparelho respiratório foi o mais acometido, seguido pelo gastrintestinal e neurológico. Não houve diferença estatisticamente significativa entre o sistema acometido e o desfecho óbito. Estes dados são coincidentes com trabalhos realizados previamente em Belo Horizonte e em outras cidades brasileiras, tendo as infecções oportunistas como as principais responsáveis pela necessidade de internação hospitalar ${ }^{413}$.

Os diagnósticos mais freqüentemente realizados das doenças oportunistas foram de pneumocistose, pneumonia comunitária, síndrome diarréica e candidiase oral. Dados apresentados por estudo conduzido no Hospital das Clínicas da UFMG em 2000 revelaram que a candidiase era o diagnóstico mais freqüente, seguido por tuberculose e pneumocistose ${ }^{13}$. Os dados aqui apresentados revelam que entre 2000 e 2007 houve importância crescente da pneumonia comunitária e redução da tuberculose como responsáveis pela necessidade de tratamento hospitalar da AIDS.

\section{AGRADECIMENTOS}

Ao Curso de Pós-Graduação em Ciências da Saúde: Infectologia e Medicina Tropical da Faculdade de Medicina da Universidade Federal de Minas Gerais e ao Pronto Atendimento do Hospital das Clínicas da Universidade Federal de Minas Gerais.

\section{REFERÊNCIAS}

1. Betz ME, Gebo KA, Barber E, Sklar P, Fleishman JA, Reilly ED, Christopher Mathews W. HIV Research Network. Patterns of diagnoses in hospital admissions in a Multistate Cohort of HIV- positive adults in 2001. Medical Care 43: III 3-14, 2005.

2. Davaro R; Thirumalai A. Life-threatening complications of HIV infections. Journal of Intensive Care Medicine 22:73-81, 2007.

3. De Brito AM, De Castilho EA, Szwarcwald CL. Regional Patterns of the temporal evolution of the AIDS epidemic in Brazil following the introduction of Antiretroviral Therapy. Brazilian Journal of Infectious Diseases 9: 9-19, 2005. 
4. Dourado I, Veras MA, Barreira D, de Brito AM. AIDS epidemic trends after the introduction of Antiretroviral Therapy in Brazil. Revista de Saúde Pública 40:9-17, 2006

5. Gebo KA, Fleishman JA, Moore RD. Hospitalizations for metabolic conditions, opportunistic infections, and injection drug use among HIV patients. Trends between 1996 and 2000 in 12 States. Journal of Acquired Immune Deficiency Syndrome; 40:609-616, 2005.

6. Hacker MA, Petersen ML, Enriquez M, Bastos FI. Highly Active Antiretroviral Therapy in Brazil: The challenge of Universal Access in a context of social inequality. Revista Panamericana de Salud Pública/Pan American Journal of Public Health 16: 78-83, 2004.

7. Huang L, Quartin A, Jones D, Havlir DV. Intensive Care of Patients with HIV infection. The New England Journal of Medicine 355: 173-181, 2006.

8. Kaplan JE, Hanson E, Dworkin MS, Frederick T, Bertolli J, Lindegren ML, Holmberg S, Jones JL. Epidemiology of Human Immunodeficiency Virusassociated opportunistic infections in the United States in the era of Highly Active Antiretroviral. Therapy. Clinical Infectious Diseases 30: s5-14, 2000.

9. Ministério da Saúde. Boletim epidemiológico AIDS. 2006. Disponível em <http:// www.aids.gov.br/final/dados/bol_aids1.htm>. acesso em: 30 out, 2007.

10. Mocroft A, Monforte A, Kirk O, Johnson MA, Friis-Moller N, Banhegyi D, Blaxhult A, Mulcahy F, Gatell JM, Lundgren JD; EuroSIDA study group. Changes in Hospital admissions across Europe: 1995-2003. Results from the EUROSIDA Study. HIV Medicine 5: 437-447, 2004.

11. Morris A, Creasman J, Turner J, Luce JM, Wachter RM, Huang L. Intensive care of Human Immunodeficiency Virus- infected patients during the Era of Highly
Active Antiretroviral Therapy. American Journal of Respiratory and Critical Care Medicine 166: 262-267, 2002.

12. Murphy EL, Collier AC, Kalisch LA, Assmann SF, Para MF, Flanigan TP, Kumar PN, Mintz L, Wallach FR, Nemo GJ; Viral Activation Transfusion Study Investigators. Highly Active Antiretroviral Therapy Decreases Mortality Morbidity in patients with advanced HIV disease. Annals of Internal Medicine 135:17-26, 2001.

13. Nobre V, Braga E, Raves A, Serufo JC, Godoy P, Nunes N, Antunes CM, Lambertucci JR. Infecções oportunistas em pacientes com AIDS internados em um hospital universitário do sudeste do Brasil. Revista do Instituto de Medicina Tropical de São Paulo 45: 69-74, 2003.

14. Nuesch R, Geigy N, Schaedler E, Battegay M. Effect of Highly Active Antiretroviral Therapy on hospitalization characteristics of HIV-infected patients. European Journal of Clinical Microbiology \& Infectious disease 21:684-687, 2002.

15. Palacios R, Hidalgo A, Reina C, de la Torre M, Márquez M, Santos J. Effect of Antiretroviral Therapy on admissions of HIV-infected patients to an Intensive Care Unit. HIV Medicine; 7: 193-196, 2006

16. Palella Jr FJ, Delaney KM, Moorman AC, Loveless MO, Fuhrer J, Satten GA, Aschman DJ, Holmberg SD. Declining morbidity and mortality among patients with advanced human immunodeficiency virus infection. HIV Outpatient Study Investigators. The New England Journal of Medicine 338: 853-860, 1998.

17. Plá Bento CA. Estudo do papel da intervenção de serviço de assistência domiciliar para pacientes co-infectados por Vírus da Imunodeficiência Humana e Tuberculose recém desospitalizados de Hospital referência em Belo Horizonte, MG sobre a interrupção das terapias anti-retroviral e tuberculostática. Dissertação de Mestrado, Universidade Federal de Minas Gerais, Belo Horizonte, 2006. 